\title{
BMJ Open Prevalence of multimorbidity among Bangladeshi adult population: a nationwide cross-sectional study
}

\author{
Nusrat Khan, ${ }^{1}$ Mahfuzar Rahman, ${ }^{1}$ Dipak Mitra, ${ }^{2}$ Kaosar Afsana ${ }^{3,4}$
}

To cite: Khan N, Rahman M, Mitra D, et al. Prevalence of multimorbidity among Bangladeshi adult population: a nationwide crosssectional study. BMJ Open 2019;9:e030886. doi:10.1136/ bmjopen-2019-030886

- Prepublication history for this paper is available online. To view these files, please visit the journal online (http://dx.doi. org/10.1136/bmjopen-2019030886).

Received 08 April 2019 Revised 24 September 2019 Accepted 15 October 2019

Check for updates

(C) Author(s) (or their employer(s)) 2019. Re-use permitted under CC BY-NC. No commercial re-use. See rights and permissions. Published by BMJ.

${ }^{1}$ Research and Evaluation Division, BRAC, Dhaka,

Bangladesh

${ }^{2}$ School of Public Health, North South University, Dhaka, Dhaka District, Bangladesh

${ }^{3}$ Health, Nutrition and Population Program, BRAC, Dhaka, Dhaka, Bangladesh

${ }^{4}$ James P Grant School of Public Health, BRAC University, Dhaka, Dhaka District, Bangladesh

Correspondence to

Dr Nusrat Khan;

nusrat.khan@brac.net

\section{ABSTRACT}

Objective This study aimed to report prevalence and evaluate the association between multimorbidity and associated risk factors in the adult population of Bangladesh.

Design A cross-sectional study was conducted using a multistage clustered random sampling strategy. Setting The study was conducted among the general population of 58 districts in Bangladesh.

Participants A total of 12338 male and female individuals aged $\geq 35$ were included for analysis in this study. Identified through a household listing conducted prior to the study, from 15297 individuals meeting the inclusion criteria, 12338 participants were included based on availability during data collection, consent and health condition.

Outcome measures Multimorbidity in terms of hypertension, diabetes, cancer, cardiovascular diseases, stroke and chronic obstructive pulmonary disease. Results Approximately $8.4 \%(95 \% \mathrm{Cl} 7.0$ to 9.7$)$ of individuals suffer from multimorbidity, of which hypertension accounted for (30.1\%) followed by diabetes (10.6\%). The mean age of the population was 58.6 (SD \pm 9.2 ) years. The prevalence of multimorbidity was lower among men (7.7\%) compared with women (8.9\%). The likelihood of having multimorbidity among obese individuals were more than double than people with normal body mass index (BMI). Physical activity protected individuals from developing multimorbidity: however, the physical activity adjusted OR was $0.5(95 \% \mathrm{Cl} 0.2$ to 1.2). After adjusting for all covariates, higher age, higher educational status, economic status, and higher BMI were found to be significantly associated with the odds of developing multimorbidity, with an overall adjusted OR of 0.02 (95\% Cl 0.01 to 0.02$)$.

Conclusion This study reported a high prevalence of multimorbidity in Bangladesh, although it explored the burden and identified risk factors considering only six chronic diseases. Further detailed exploration through longitudinal studies considering a wider range of diseases is needed to document the actual burden, develop effective preventive measures and clinical guidelines to improve the quality of life of the population.

\section{INTRODUCTION}

Multimorbidity is becoming an emergent global health concern. It is commonly defined as the presence of two or more
Strengths and limitations of this study

First large-scale national-level study in Bangladesh reporting the prevalence of multimorbidity with rural and urban area coverage. It also reports the current national-level prevalence of major chronic diseases.

- The study relied on the self-reported chronic disease data and also cross-checked prescriptions written by registered physicians.

- While the study collected and analysed data at the national level, the rural sample is over-represented.

- We took into account only six major predetermined chronic diseases; understandably, when considering the full range of possible diseases, the proportion would be higher.

- Given its cross-sectional design, the study cannot demonstrate a causal relationship, only association.

chronic diseases in an individual. ${ }^{1}$ Multimorbidity presents several challenges to any healthcare system since most are generally focused on treating single diseases rather than multiple coexisting conditions. More common among the elderly population, the growing demand for healthcare needs to deal with multiple non-communicable diseases/multimorbidity and associated polypharmacy is putting considerable strain on healthcare systems around the world. ${ }^{2}$ This certainly adds to the already challenging burden of addressing chronic diseases. At the patient level, coexisting multiple diseases in an individual increase financial constraints and also complicate life in various ways-the decreasing quality of life and productivity, adherence to complex medication regimens, multiple specialised healthcare needs and often massive changes in lifestyle.

Multimorbidity adversely affects the health and well-being of the individual in many ways. First, it leads to lower disability-adjusted life years. Patients suffering from multimorbidity have a high treatment burden in terms of understanding and self-managing diseases. ${ }^{3}$ Second, it is associated with decreased quality of life and increased healthcare utilisation, 
including higher incidences of emergency admissions and referral points. Third, e management of multimorbidity with drugs is often complex, resulting in polypharmacy and its associated risks. ${ }^{4}$ This includes primary and secondary care outpatient visits and often multiple hospital admissions. Patients from lower socioeconomic groups are generally even more burdened given the high financial costs of dealing with long-term diseases. In health system aspect, managing patients with multimorbidity overall is found to be taxing for the patients and also for practitioners because of complex regimens, multiple entry points and referrals. ${ }^{4-7}$ As a result, there is a growing consensus that the increasing prevalence of multimorbidity is likely to put challenges on healthcare systems and ultimately healthcare costs, making poor people even poorer. ${ }^{8}$

Mounting evidence suggests that sociodemographic factors are associated with multimorbidity. ${ }^{9}$ Furthermore, different studies have found lifestyle factors including smoking, obesity, unhealthy diet and alcohol to be linked with multimorbidity as well. ${ }^{9}{ }^{10}$ Most of the studies and guidelines on the management of long-term conditions have traditionally been focused on single diseases rather than multimorbidity. Evidence on population-based estimates and risk factors of multimorbidity are also not readily available globally, which makes future planning a challenge. ${ }^{6}$

So far, most of the population-based studies adopted the WHO-recommended definition of multimorbidity-'coexisting multiple chronic diseases in an individual' to quantify multimorbidity burden, although a list of recommended diseases has not been specified. ${ }^{1} \mathrm{~A}$ large-scale study in Canada documented that the prevalence of multimorbidity among Ontarians rose from $17.4 \%$ in 2003 to $24.3 \%$ in 2009 , denoting a $40 \%$ escalation. This increase over time was evident across all age groups. Another study in Catalonia, Spain reported that, when all chronic conditions were included in the analysis, almost $50 \%$ of the adult urban population had multimorbidity. ${ }^{11-13}$ In Portugal, multimorbidity was present in $72.7 \%$ of the population. In China, the prevalence is found to be above $50 \%$ in the elderly population. ${ }^{14}$ In the $\mathrm{UK}$, the number of people with three or more long-term conditions is estimated to have risen from 1.9 million in 2008 to 2.9 million in $2018 .^{9}$ This wide variation in terms of reported prevalence is mainly due to the number of diseases included in the measurement of multimorbidity.

Multimorbidity is a growing area of research in the western world, but there is limited evidence on the burden among developing countries. South Asians have already been characterised as an inherently high-risk group for developing cardio-metabolic and other chronic diseases, and thus multimorbidity is thought to be significantly prevalent in these populations. ${ }^{2}$ Bangladesh, being a developing country in South-East Asia and struggling with the double burden of infectious as well as non-communicable diseases (NCDs), has not yet addressed this health issue at the national level, although it can be a challenge for the healthcare system in near future, given the rising size of older populations. There is also very limited documentation on the status of multimorbidity, with no nationally representative large-scale data. Many chronic conditions lead to a multimorbid status associated with increasing age. As per the WHO Non-Communicable Disease (NCD) Report 2011, more than 52\% of deaths in Bangladesh are caused by different NCDs and more than $27 \%$ are due to cardiovascular causes. In the NCD risk factors survey conducted in 2010 by the Ministry of Health and Family Welfare, almost $99 \%$ of the Bangladeshi population had at least one NCD risk factor; out of those, $29 \%$ had three or more risk factors. A recent study projected that by 2025, the death rate from cardiovascular diseases will increase 21 times. ${ }^{15}$ This evidence denotes the importance of NCDs in Bangladesh healthcare planning. While a study on the rural elderly population reported a $53.7 \%$ prevalence of multimorbidity in Bangladesh, ${ }^{16}$ a large-scale nationally representative study is of great importance to generate evidence on current status (prevalence, incidence and risk factors). Therefore, the purpose of our study was to capture the current burden and risk factors of multimorbidity in Bangladesh including participants from a wider age group ( $\geq 35$ years).

\section{METHODOLOGY}

BRAC (Building Resources Across Communities), the world's number 1 non-government organisation, has been a pioneer in its health initiatives since its inception. Through its flagship health intervention, health nutrition and population programme (HNPP), it has come up with a comprehensive healthcare package to assist the vulnerable population of the country at an affordable cost. A large-scale cross-sectional study was conducted in 2014 to explore the status of selected healthcare indicators (in line with the Strategic Partnership Arrangement of BRAC) in HNPP working areas of BRAC. Information on NCDs as well as chronic diseases and multimorbidity was extremely important, as NCD status exploration was one of the key focus. The data were collected from a nationwide sample covering both rural and urban areas.

\section{Study design and population}

The cross-sectional study was conducted using a multistage clustered random sampling strategy. Details about the data collection process can be found elsewhere. ${ }^{17}$ Put briefly, we collected information from 11428 households, with a response rate of $94.9 \%$. In the first stage of sampling, 210 enumeration areas (EAs) were selected randomly with probability proportional to EA size. This included 180 EAs from rural areas and 30 EAs from urban slums. An EA is a union (rural areas) or ward (urban slums) - the lowest administrative unit in Bangladesh. A union is defined as a collection of a small number of villages, whereas a ward is typically a collection of villages and/or slums. A slum is defined as a cluster of compact settlements of five or more households. A complete list of 
Respondent selection:

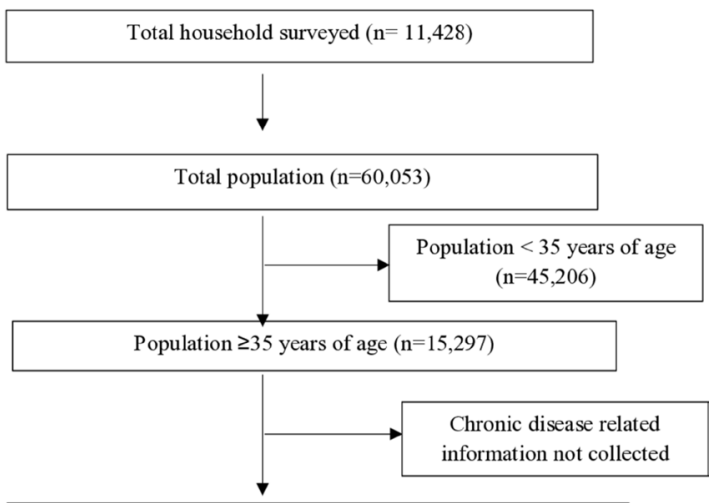

Chronic disease related information collected $(n=12,338)$

Figure 1 Study participant selection.

unions and wards was included in the sampling frame for the first stage of sampling, which was collected from the most recent Population and Housing Census of Bangladesh. ${ }^{18}$ In the second stage of sampling, starting from the north-west corner of an EA with a systematic random sample of five households, an average of 54 households per EA were selected to provide statistically reliable estimates for rural areas and urban slums separately. Information was collected from all the men and women aged $\geq 35$ years residing in the selected households, with $80.9 \%$ complete responses (figure 1).

\section{Data collection procedures and tools}

Recruitment of respondents for the study required the creation of a study base for systematic sampling based on demographic characteristics and BRAC programme interventions. To recruit eligible survey respondents from the household census, eligible respondents (willing to participate, not critically ill and $<35$ years old) were identified. The household census was carried out using a structured questionnaire between 22 August and 5 September 2015. Individual data were collected from 11 October 2015 to 6 January 2016 through face-to-face interviews with a pretested structured questionnaire from 58 out of 64 districts in Bangladesh. The questionnaire was installed in tablet personal computers using open data kit (ODK V.1.4.5) software; data collection was carried out by trained interviewers (figure 1 ).

The required sample size was calculated for an observed rate, associated margins of error (5\%) and a non-response rate. A sample size of 15297 was found to be sufficient. The sample size was calculated considering a $5 \%$ statistical significance and a design effect of $1.5 \%$ and $5 \%$ non-response rate. Among them, 12338 people provided anthropometric measurements, and their blood pressure and urinary glucose were assessed. Chronic disease data were self-reported, and prescription verified by trained enumerators.

A detailed questionnaire was developed, and the tool was pre-tested (150 samples) in both rural and urban areas by trained enumerators before the commencement of the study. A thorough review by the lead researchers resulted in required modifications of the questions. A total of 110 skilled interviewers (with prior experience in conducting large-scale healthcare surveys) were recruited for data collection.

Respondents were ensured of the confidentiality of any information provided. The respondents were informed that they had the full right to withdraw their participation at any time during the interview. Data were stored in BRAC head office servers to which only the researchers of the group had access. Critically ill patients were excluded from the study.

\section{Measurement of variables}

The primary outcome variable for this study is multimorbidity, which is when an individual suffers from two or more of the following NCDs: hypertension, diabetes, chronic obstructive pulmonary disease (COPD), cancer, heart diseases and stroke. At first, history was taken from the participants. Later, prescriptions were checked for validity of diagnosis by a registered physician. To measure the presence of hypertension, we included respondents who were previously diagnosed by a registered doctor, were taking anti-hypertensive medication or were found to be hypertensive (systolic $\geq 140 \mathrm{~mm} \mathrm{Hg}$ or diastolic $\geq 90 \mathrm{~mm} \mathrm{Hg}$ ) by measurement of blood pressure at the time of the interview. For diabetes, we included people who had been diagnosed by doctors as diabetic, were on medication or tested positive on a urinary strip test at the time of interview. Other diseases were confirmed by asking the respondents whether they had been diagnosed by a registered physician, as well as by checking the available prescriptions.

The list of potential risk factors of multimorbidity was based on literature about the risk factors of different chronic diseases. The assumption was that that the risk factors will be similar for multiple chronic diseases combined. These include sociodemographic characteristics (age, sex, educational status and economic status), as well as health and lifestyle-related factors (physical (in) activity, obesity, tobacco use, sleeping habit and dietary diversity).$^{79}$ These data were collected through face-toface interviews with respondents using a questionnaire. Variables BMI (underweight, $<18.5 \mathrm{~kg} / \mathrm{m}^{2}$; normal, $18.5-24.9 \mathrm{~kg} / \mathrm{m}^{2}$; overweight, $25.0-29.9 \mathrm{~kg} / \mathrm{m}^{2}$; obese, $\geq 30.0 \mathrm{~kg} / \mathrm{m}^{2}$ ) and hypertension were categorised and calculated using standardised WHO guidelines. Daily sleeping time of the participants was measured by the standard average sleeping hour of 8 hours as per participant history taking.

\section{Statistical analysis}

We first estimated the prevalence of multimorbidity in the study population using descriptive statistics. Distributions of potential risk factors were compared between respondents with and without multimorbidity using t-tests for continuous variables and $\chi^{2}$ test of independence for 
Table 1 Prevalence of chronic diseases individually and clusters

\begin{tabular}{lc}
\hline & $\begin{array}{l}\text { Prevalence } \\
\text { (n=12338) }\end{array}$ \\
\cline { 2 - 2 } Variable & $\mathbf{n}(\%)$ \\
\hline Hypertension & $3754(30.1)$ \\
\hline Diabetes & $1310(10.6)$ \\
Cardiovascular diseases (CVDs) & $391(3.1)$ \\
\hline Cancer & $15(0.1)$ \\
Stroke & $219(1.8)$ \\
\hline Chronic obstructive pulmonary disease & $116(0.9)$ \\
(COPD) & \\
\hline Having only one physical condition & $3534(28.7)$ \\
\hline Multimorbidity (having two or more physical & $1031(8.4)$ \\
conditions) & \\
\hline Hypertension, diabetes, CVDs & $76(0.6)$ \\
\hline Hypertension, diabetes, stroke & $45(0.4)$ \\
\hline Hypertension, diabetes, cancer & $1(0.0)$ \\
\hline Hypertension, diabetes, COPD & $14(0.1)$ \\
\hline Hypertension, CVDs, stroke & $40(0.3)$ \\
\hline Diabetes, CVDs, stroke & $21(0.2)$ \\
\hline Hypertension, diabetes, CVDs, stroke & $18(0.6)$ \\
\hline
\end{tabular}

categorical variables. The association between potential risk factors and multimorbidity was estimated by crude and adjusted ORs using bi-variate and multivariate logistic regression models respectively. We used 'robust' option to adjust for complex sampling design. An association was considered significant if $p$ value was $<0.05$. Crude OR was used to identify the association of each of the independent variables while the adjusted OR (adjusted for confounding considering sociodemographic and lifestyle factors such as age, sex, BMI, physical activity, fruit, and fish and meat intake) identified the association of individual factors with the outcome measurement $p$ value $<0.05$. The fitness of the final model was tested using Hosmer-Lemeshow goodness-of-fit test ( $p$ value was not significant). All analyses were done using STATA V.12. ${ }^{19}$

\section{Patient and public involvement}

No patient was involved in this study. They were recruited after the initial household census. They were not involved with the study design, recruitment or conduction of the study. Consent was received for further publication. We are thankful for their consent, participation and co-operation.

\section{RESULTS}

Table 1 illustrates the prevalence of chronic diseases individually and in clusters. The prevalence of individual diseases among study participants was as follows: hypertension, $30.1 \%$; diabetes, $10.6 \%$; cardiac diseases, $3.2 \%$; stroke, $1.8 \%$; COPD, $0.9 \%$; cancer, $0.2 \%$. More than $60 \%$ of patients with diagnosed cardiovascular disease, cancer, stroke and COPD were on regular medication. In terms of multimorbidity, $8.4 \%$ of the study population presented two or more of the aforementioned diseases.

We next explored the association between multimorbidity and different demographic variables, as well as other risk factors (table 2). The mean age of individuals suffering from multimorbidity is 58.5 years. Women present a higher prevalence of multimorbidity than men ( $8.9 \%$ vs $7.7 \%, \mathrm{p}=0.021)$. The probability of multimorbidity among tobacco users or people who take extra salt with food is around $8.1 \%$.

In table 3 , we show how, within the study population, older people and women are significantly at higher odds of developing multimorbidity both in the crude and adjusted model. People belonging to the top two wealth quintiles and with higher educational status have a significantly higher probability of having developed multimorbidity than poorer and less educated respondents. Odds of multimorbidity in people with higher BMI $(\geq 25)$ were 2.10 times the odds in people with normal BMI. The OR comparing physically active people with those who are not active at all is 0.5 (95\% CI 0.2 to 1.3$)$.

\section{DISCUSSION}

Multimorbidity is a less explored area in healthcare research compared with individual diseases, which are typically explored both clinically and epidemiologically. The recent Sustainable Development Goals (SDGs) suggest that the post-2015 agenda will be substantially influenced by a focus on 'health in all ages', including Target 3.8 which aims to achieve universal health coverage. The realisation of these goals and other wider development targets will require multiple morbidities to be addressed. ${ }^{20}$ Therefore, with an increasing burden of chronic diseases worldwide, individuals suffering from multiple chronic diseases are also emerging as a healthcare priority. ${ }^{11}$ This is the first study (to our knowledge) reporting multimorbidity burden in Bangladesh conducted both in urban and rural areas, although results cannot be compared as there has not yet been any other large-scale study among the general population nor any systematic review or meta-analysis on the entire South Asia reporting the pooled prevalence and risk factors. Prevalence of multimorbidity $(8.4 \%)$ counted within only six diseases is quite high. We adopted the commonly used WHO definition, but the lack of data on a similar context and a similar population, as well as the absence of a consensual definition, make our findings less comparable. As we used appropriate sampling methods and/or calculation, our large population-based cross-sectional data were powerful enough to calculate the prevalence of chronic diseases and multimorbidity prevalence among the Bangladeshi population. A study such as this one contributes to generate evidence and inform healthcare policy-makers for timely action and preparedness. 
Table 2 Relationship of multimorbidity with demographic variables

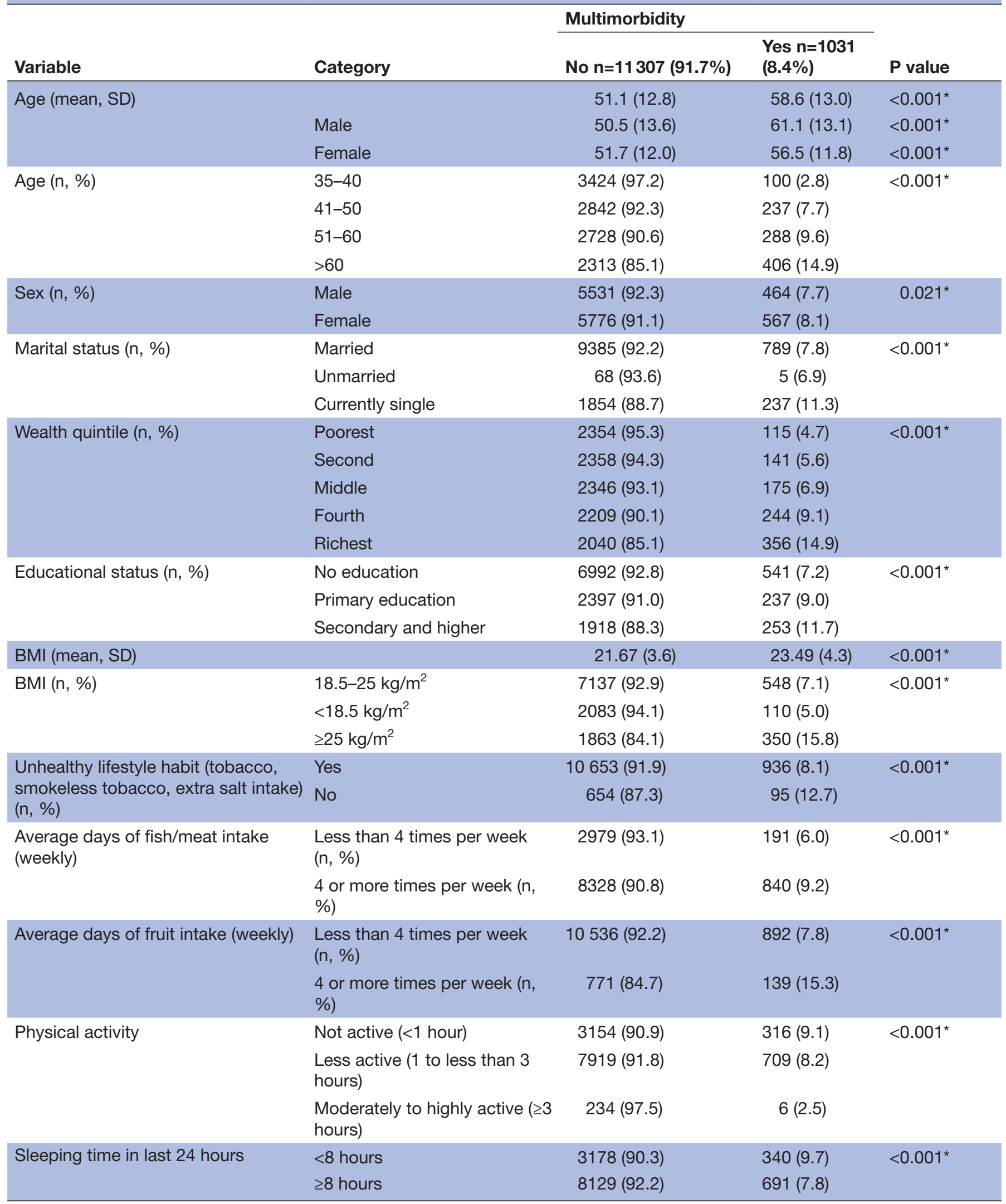

${ }^{*} \chi^{2}$ test significant $p$ value $\leq 0.05$

BMI, body mass index. 
Table 3 Crude and adjusted OR for the risk factors associated with multimorbidity

\begin{tabular}{|c|c|c|c|c|}
\hline Variable & cOR $95 \% \mathrm{Cl}$ & aOR & $P$ value & $95 \% \mathrm{CI}$ \\
\hline Constant & & 0.02 & $<0.001^{*}$ & 0.01 to 0.02 \\
\hline \multicolumn{5}{|l|}{ Age } \\
\hline $41-50$ & 2.82 .2 to 3.6 & 3.3 & $<0.001^{*}$ & 2.6 to 4.3 \\
\hline $51-60$ & 3.62 .9 to 4.5 & 4.9 & $<0.001^{*}$ & 3.8 to 6.2 \\
\hline \multicolumn{5}{|l|}{ Sex } \\
\hline Male & 1.0 & 1.0 & & \\
\hline Female & 1.21 .0 to 1.3 & 1.2 & $0.003^{*}$ & 1.1 to 1.4 \\
\hline \multicolumn{5}{|l|}{ Wealth quintile } \\
\hline Poorest & 1.0 & 1.0 & & \\
\hline Fourth & 2.31 .8 to 2.9 & 1.5 & $0.002^{*}$ & 1.2 to 1.9 \\
\hline Richest & 3.62 .9 to 4.4 & 2.1 & $<0.001^{*}$ & 1.6 to 27 \\
\hline \multicolumn{5}{|l|}{ Educational status } \\
\hline No education & 1.00 & 1.00 & & \\
\hline Primary education & 1.91 .1 to 1.5 & 1.5 & $<0.001^{*}$ & 1.3 to 1.8 \\
\hline Secondary and higher & 1.71 .5 to 2.0 & 1.8 & $<0.001^{*}$ & 1.6 to 2.3 \\
\hline \multicolumn{5}{|l|}{ BMI } \\
\hline $18.5-25$ & 1.0 & 1.0 & & \\
\hline$<18.5$ & 0.90 .6 to 0.8 & 0.6 & $<0.001^{*}$ & 0.5 to 0.8 \\
\hline
\end{tabular}

Average days of meat/fish intake (weekly)

\begin{tabular}{|c|c|c|c|c|}
\hline$<4$ times & 1.0 & 1.0 & & \\
\hline$\geq 4$ times & 1.61 .3 to 1.9 & 0.1 & 0.980 & 0.8 to 1.2 \\
\hline \multicolumn{5}{|l|}{$\begin{array}{l}\text { Average days of fruit intake } \\
\text { (weekly) }\end{array}$} \\
\hline$<4$ times & 1.0 & 1.0 & & \\
\hline$\geq 4$ times & 2.51 .8 to 2.6 & 1.3 & $0.018^{*}$ & 1.0 to 1.6 \\
\hline Less active & 0.90 .8 to 1.0 & 1.1 & 0.291 & 0.9 to 1.3 \\
\hline Moderately to highly active & 0.30 .1 to 0.6 & 0.5 & 0.152 & 0.2 to 1.3 \\
\hline
\end{tabular}

${ }^{*}$ Significant $p$ value $\leq 0.05$.

aOR, adjusted OR; cOR, crude OR.

Several studies have been conducted on major NCD status and their risk factors, many of which are assumed to be similar to multimorbidity. Most of the previous studies in the western population have shown that low socioeconomic status and less education are positively and specifically associated with multimorbidity. ${ }^{213}$ Nevertheless, our study finds the opposite results, which are also statistically significant. For higher educational status, adjusted OR (aOR) was 1.8 (95\% CI 1.6 to 2.3), and for the richest wealth quintile, aOR was 2.1 (95\% CI 1.6 to 2.7). This could owe to the fact that, being a lower-middle-income country, people with a higher economic status in Bangladesh have access to more food and education. This is consistent with findings from a recent systematic review, reporting that individuals of low socioeconomic status in low-income and lower-middle-income countries are more likely to consume a less healthy diet (eg, less fruit, vegetables, fish and fibre, but more meat). Whereas, individuals of higher socioeconomic status tend to be more physically inactive, thus increasing the prevalence of NCDs. ${ }^{21}$ As we 
already know, lifestyle factors are closely associated with chronic diseases and multimorbidity risk. We can therefore say that since people of higher economic status have more access to food and also traditionally live a sedentary lifestyle, this leads them to develop more NCD risk factors and ultimately multimorbidity. ${ }^{22}$ Identifying higher age and being female as a significant determinant of multimorbidity is consistent with existing literature. ${ }^{27}$

On the contrary, in developed countries, epidemiological evidence is scarce. South Asians have already been characterised as a genetically high-risk group for developing cardio-metabolic and other chronic diseases, resulting in a higher incidence of multimorbidity within the population. ${ }^{2}$ The risk factors of multimorbidity identified in our study are similar to the reported risk factors of individual chronic diseases in various analyses. As in many other studies, BMI showed a significant association with multimorbidity. ${ }^{7}$ Dozens of epidemiological studies have reported findings on the association between fruit and vegetable intake on one hand, and the risk of cardiovascular disease and other NCDs on the other. ${ }^{22}{ }^{23}$ Our study also corroborates that a higher intake of fish and meat is significantly associated with multimorbidity in the crude analysis, but not in the adjusted model. Increased intake of fruits and vegetables were found to be protective factors against developing multimorbidity, but after adjusting for covariates, this result was not significant. Among other lifestyle factors, increased physical activity and a higher average daily sleeping time were found to be protective factors against developing multimorbidity. ${ }^{13} 14$

Although most of the evidence on multimorbidity is limited to the elderly population globally, this study is unique in the sense that it covers a wider age range. Our study reports that the odds of developing multimorbidity are higher among those on the older cohort compared with the middle-age group (35-40). Even in the age groups 41-50 and 51-60, the odds of developing multimorbidity are respectively 2.8 and 3.6 times higher than among people under 40 years of age. This suggests that people develop multimorbidity even during active and productive years of life, which can cause excess healthcare costs from earlier years in life. In a context of less developed health infrastructure and of an inadequacy of human resources, this evidence on the burden of multimorbidity in Bangladesh warrants further exploration from various perspectives, including further research as well as the development of policies to tackle the burden. Therefore, population-based data on multimorbidity is an urgent need to get the actual scenario of the burden and further develop strategies to prepare the healthcare systems. Otherwise, with the ageing population and everrising chronic disease burden globally, healthcare systems shall not be prepared to tackle this healthcare challenge.

\section{LIMITATIONS}

The study has several noteworthy points, and the findings raise new questions. Markedly, it shows that among 12338 individuals in Bangladesh, nearly $8.4 \%$ were suffering from multimorbidity. This proportion can be considered a lower bound estimate considering we only took into account six pre-determined clinical diseases; without a doubt, when considering the full range of possible diseases, the proportion would be higher. ${ }^{23}$ Several potential limitations, however, should also be noted. First, we only considered six diseases, and only hypertension and diabetes data were measured,while the remaining four were self-reported by the respondents based on the previous diagnosis by the physicians, which might include selection biases. Second, the validity of the physician reported diagnosis and laboratory diagnosis should have been explored more precisely for conclusiveness. Third, it would have been desirable to have standard-scale data of physical activity. Without a scaling guideline, comparisons against other findings are not possible. Fourth, our findings only draw on data from those areas in which BRAC has coverage. Since BRAC only covers $80 \%$ of the whole population, the results cannot strictly be considered representative of Bangladesh. The rural sample might also be over-represented (93\%). Meanwhile, the urban data are generalisable to only the urban slum areas and therefore it may not be wise to generalise for the urban population of Bangladesh; that is, we may be under-reporting the prevalence of multimorbidity among the urban population. A precise nationally representative sampling strategy would have been desirable to report the actual nationally representative data on multimorbidity burden. ${ }^{13}$ Fifth, considering the cross-sectional design of our study, the current results do not demonstrate a causal relationship, but rather associations which suggest possible effects. A well-designed cohort study with prospective data is essential to predict mortality and report incidence. ${ }^{24}$ Sixth, we have only adjusted for potential confounding variables for which data were available during this cross-sectional study. Lastly, a combination of population-based and hospitalbased data would be more representative and might indicate a higher prevalence. ${ }^{15}$ Despite its many limitations, the major strength of the study is that we did not rely on self-reported chronic disease data, and instead included those that were diagnosed by a registered physician.

\section{CONCLUSION}

This study identifies the prevalence of multimorbidity among Bangladeshi individuals who are undergoing an epidemiological transition. It is comparable with many developing countries and thus highlights the importance of recognising associated risk factors, especially among young adults and also in the elderly. A prevalence of nearly $9 \%$ in a nationwide sample is alarming. The lack of a consensual definition of multimorbidity may also contribute to underestimation or overestimation. We recommend that an international clinical guideline with an approved range of diseases should be developed to serve the purpose, which would also aid in monitoring progress towards the fulfilment of the United Nations' 
SDGs. As for Bangladesh, there is a need for a detailed national-level exploration including the rural and urban population in a larger scale to document the current prevalence, incidence and specific risk factors associated with multimorbidity. This would help inform policy-makers and further develop preventive strategies and clinical guidelines.

Acknowledgements The Research and Evaluation Division of BRAC acknowledges with gratitude the commitment of the BRAC to its research effort. We are also thankful to the health unit of the division for their continuous contribution. We acknowledge Roberto Zedillo Ortega from University of Cambridge for kindly reviewing the manuscript for English correction and giving his valuable feedback.

Contributors Conceptualisation: NK, MR, KA. Formal analysis: NK, MR. Investigation: NK, MR, DM. Methodology: NK, MR, DM. Supervision: MR, KA. Writing original draft: NK. Writing — review and editing: NK, MR, KA, DM. All authors read and approved the final manuscript.

Funding This research activity was funded by the Strategic Partnership Arrangement(SPA) between BRAC, the UK Department for International Development(DFID) and the Australian Department of Foreign Affairs and Trade(DFAT) in 2015. The funders had no role in the design, analysis or writing ofthis manuscript.

Competing interests None declared.

Patient consent for publication Obtained.

Ethics approval The ethical approval was obtained from the Bangladesh Medical Research Council (BMRC). The purpose of the study was described to the participants. Both verbal and written consent was provided by participants prior to the census and main interview and the respondents were ensured of the confidentiality of information provided.

Provenance and peer review Not commissioned; externally peer reviewed.

Data availability statement Data available on request.

Open access This is an open access article distributed in accordance with the Creative Commons Attribution Non Commercial (CC BY-NC 4.0) license, which permits others to distribute, remix, adapt, build upon this work non-commercially, and license their derivative works on different terms, provided the original work is properly cited, appropriate credit is given, any changes made indicated, and the use is non-commercial. See: http://creativecommons.org/licenses/by-nc/4.0/.

\section{REFERENCES}

1 Afshar S, Roderick PJ, Kowal P, et al. Multimorbidity and the inequalities of global ageing: a cross-sectional study of 28 countries using the world health surveys. BMC Public Health 2015;15:776.

2 Coventry PA, Lovell K, Dickens C, et al. Update on the collaborative interventions for circulation and depression (COINCIDE) trial: changes to planned methodology of a cluster randomized controlled trial of collaborative care for depression in people with diabetes and/ or coronary heart disease. Trials 2013;14:136.

3 Fortin M, Lapointe L, Hudon C, et al. Multimorbidity and quality of life in primary care: a systematic review. Health Qual Life Outcomes 2004;2:51.

4 Dumbreck S, Flynn A, Nairn M, et al. Drug-disease and drug-drug interactions: systematic examination of recommendations in 12 UK national clinical guidelines. BMJ 2015;350:h949.
5 Liu LM. Deprescribing: an approach to reducing polypharmacy in nursing home residents. J Nurse Pract 2014;10:136-9.

6 Moffat K, Mercer SW. Challenges of managing people with multimorbidity in today's healthcare systems. BMC Fam Pract 2015;16:129.

7 Ahmadi B, Alimohammadian M, Yaseri M, et al. Multimorbidity. Medicine 2016;95:e2756.

8 Thavorn K, Maxwell CJ, Gruneir A, et al. Effect of socio-demographic factors on the association between multimorbidity and healthcare costs: a population-based, retrospective cohort study. BMJ Open 2017;7:e017264

9 Li J, Green M, Kearns B, et al. Patterns of multimorbidity and their association with health outcomes within Yorkshire, England: baseline results from the Yorkshire health study. BMC Public Health 2016;16:649.

10 Wallace E, Salisbury C, Guthrie B, et al. Managing patients with multimorbidity in primary care. BMJ 2015;350:h176.

11 Hughes LD, McMurdo MET, Guthrie B. Guidelines for people not for diseases: the challenges of applying UK clinical guidelines to people with multimorbidity. Age Ageing 2013;42:62-9.

12 Koné Pefoyo AJ, Bronskill SE, Gruneir A, et al. The increasing burden and complexity of multimorbidity. BMC Public Health 2015;15:415.

13 Violan C, Foguet-Boreu Q, Flores-Mateo G, et al. Prevalence, determinants and patterns of multimorbidity in primary care: a systematic review of observational studies. PLoS One 2014:9:e102149.

14 Prazeres F, Santiago L. Prevalence of multimorbidity in the adult population attending primary care in Portugal: a cross-sectional study. BMJ Open 2015;5:e009287.

15 Non-Communicable Disease Risk Factor Survey Bangladesh 2010 Bangladesh Society of Medicine [Internet], 2011. Available: https:// www.who.int/ncds/surveillance/steps/2010_STEPS_Report_ Bangladesh.pdf [Accessed 22 Jul 2019].

16 Khanam MA, Streatfield PK, Kabir ZN, et al. Prevalence and patterns of multimorbidity among elderly people in rural Bangladesh: a cross-sectional study. J Health Popul Nutr 2011;29:406-14.

17 Khanam F, Hossain MB, Mistry SK, et al. Prevalence and risk factors of cardiovascular diseases among Bangladeshi adults: findings from a cross-sectional study. J Epidemiol Glob Health 2019;9:176-84.

18 Bangladesh Bureau of Statistics (BBS). Bangladesh population and housing census 2011 national report, [Internet], 2011. Available: http://203.112.218.66/WebTestApplication/userfiles/Image/BBS/ Socio_Economic.pdf [Accessed 22 Jul 2019].

19 StataCorp. Stata statistical software: release 12. College Station, TX: StataCorp LP, 2011.

20 The Academy of Medical Sciences. Multimorbidity: a priority for global health research [Internet], 2018. Available: https://acmedsci. ac.uk/file-download/82222577 [Accessed 5 Jul 2019].

21 Allen L, Williams J, Townsend N, et al. Socioeconomic status and non-communicable disease behavioural risk factors in low-income and lower-middle-income countries: a systematic review. Lancet Glob Health 2017;5:e277-89.

22 Bazzano LA, He J, Ogden LG, et al. Fruit and vegetable intake and risk of cardiovascular disease in US adults: the first national health and nutrition examination survey epidemiologic follow-up study. Am J Clin Nutr 2002;76:93-9.

23 Jakovljević M, Ostojić L. Comorbidity and multimorbidity in medicine today: challenges and opportunities for bringing separated branches of medicine closer to each other. Psychiatr Danub 2013;25 Suppl 1:18-28.

24 BELIEVE (Bangladesh Longitudinal Investigation of Emerging Vascular Events Study) [Internet]. Available: https://www.phpc.cam. ac.uk/ceu/international-vascular-health/believe/ [Accessed $20 \mathrm{Jul}$ 2019]. 